OPEN ACCESS

Edited by:

Maria S. Garcia-Gutierrez, Miguel Hernández University of Elche,

Spain

Reviewed by:

Bhaskar Roy,

University of Alabama at Birmingham,

United States

Reiji Yoshimura,

University of Occupational and

Environmental Health Japan, Japan

*Correspondence: Alexandra S. Troyan troian@zmapo.edu.ua

Oleg A. Levada

olevada@zmapo.edu.ua

Specialty section: This article was submitted to Molecular Psychiatry, a section of the journal Frontiers in Psychiatry

Received: 27 November 2019 Accepted: 24 July 2020

Published: 13 August 2020

Citation:

Troyan AS and Levada OA (2020) The Diagnostic Value of the Combination of Serum Brain-Derived Neurotrophic

Factor and Insulin-Like Growth Factor-1 for

Major Depressive Disorder Diagnosis and Treatment Efficacy.

Front. Psychiatry 11:800. doi: 10.3389/fpsyt.2020.00800

\section{The Diagnostic Value of the} Combination of Serum Brain-Derived Neurotrophic Factor and Insulin-Like Growth Factor-1 for Major Depressive Disorder Diagnosis and Treatment Efficacy

\author{
Alexandra S. Troyan ${ }^{*}$ and Oleg A. Levada *
}

Psychiatry Course, State Institution "Zaporizhzhia Medical Academy of Postgraduate Education Ministry of Health of Ukraine", Zaporizhzhia, Ukraine

Background: Last decades of psychiatric investigations have been marked by a search for biological markers that can clarify etiology and pathogenesis, confirm the diagnosis, screen individuals at risk, define the severity, and predict the course of mental disorders. In our study, we aimed to evaluate if BDNF and IGF-1 serum concentrations separately and in combination might be used as biomarkers for major depressive disorder (MDD) diagnosis and treatment efficacy and to evaluate the relationships among those proteins and clinical parameters of MDD.

Methods: Forty-one MDD patients (according to DSM-5) and 32 healthy controls $(\mathrm{HC})$ were included in this study. BDNF and IGF-1 serum concentrations, psychopathological (MADRS, CGI) and neuropsychological parameters (PDQ-5, RAVLT, TMT-B, DSST), functioning according to Sheehan Disability Scale were analyzed in all subjects at admission and 30 MDD patients after 8 weeks of vortioxetine treatment. Correlational analyses were performed to explore relationships between BDNF and IGF-1 and clinical characteristics. AUC-ROCs were calculated to determine if the value of serum BDNF and IGF-1 levels could serve for MDD diagnosis.

Results: MDD patients had significantly lower serum BDNF $(727.6 \pm 87.9$ pg/ml vs. 853.0 $\pm 93.9 \mathrm{pg} / \mathrm{ml})$ and higher serum IGF-1 levels $(289.15 \pm 125.3 \mathrm{ng} / \mathrm{ml}$ vs. $170.2 \pm 58.2 \mathrm{ng} /$ $\mathrm{ml}$ ) compared to HC. Significant correlations were obtained between BDNF levels and MDD status, depressive episode (DE) severity, precipitating factors, executive functions disruption (TMT-B, RAVLT immediate recall scores) and all subdomains of functioning. As for IGF-1, correlations were found between IGF-1 level and MDD status, DE severity, number and duration of DE, parameters of subjective and objective cognitive functioning (PDQ-5, RAVLT, TMT-B, DSST scores), and all subdomains of functioning. The associations between IGF-1 concentrations and cognitive tests' performance were stronger than those of BDNF. Separately both BDNF and IGF-1 demonstrated good 
discriminating ability for MDD diagnosis with AUC of 0.840 and 0.824 , respectively. However, the combination of those neurotrophins had excellent diagnostic power to discriminate MDD patients from $\mathrm{HC}$, providing an AUC of 0.916. Vortioxetine treatment significantly increased BDNF and attenuated IGF-1 serum concentrations, improved all psychopathological and neuropsychological parameters and functioning.

Conclusions: The combination of IGF-1 and BDNF might be considered as a diagnostic combination for MDD.

Keywords: major depressive disorder, insulin-like growth factor-1, brain-derived neurotrophic factor, biomarkers, cognitive functions, vortioxetine

\section{INTRODUCTION}

The last decades of psychiatric investigations have been marked by a search for biological markers that can clarify etiology and pathogenesis, confirm a diagnosis, screen individuals at risk, define the severity, and predict the course of mental disorders (1-3). With respect to major depressive disorder (MDD), abnormal neuroplasticity in cerebral regions, responsible for emotional and cognitive processing, is considered to be one of the key pathogenic mechanisms $(4,5)$ that potentially have a biomarker value. It is associated with alterations in the expression of neurotrophic factors, such as brain-derived neurotrophic factor (BDNF), neurotrophin-3, neurotrophin-4/5, nerve growth factor, insulin-like growth factor (IGF-1), etc. (6-8). The vital role of the neurotrophins is explained by their involvement in the processes of neuronal growth, differentiation, maturation, and survival, synaptic transmission, an equilibrium between neuroregeneration and neurodegeneration, as well as memory formation (9). Therefore, there is reason to believe that two proteins-BDNF and IGF-1-can be used as marker molecules for the MDD diagnosis and therapy effectiveness $(7,8)$.

Recent studies have shown lower BDNF concentrations in the brains of suicidal MDD persons $(10,11)$. Further, postmortem studies of MDD individuals revealed an association between the diminished BDNF expression in hippocampal areas and a decrease in the volume of these anatomical structures (12). According to meta-analyses, serum and plasma BDNF reductions have been noted in antidepressant-free individuals with MDD as compared to healthy subjects (13-15). The decreased peripheral BDNF levels normalized in reprisal for several interventions [e.g., antidepressants $(16,17)$, electroconvulsive therapy $(18,19)$, aerobic exercise (20)]. Thus, we can reasonably assume that the decline in peripheral (plasma/serum) BDNF levels reflects its reduced expression in the brain and could serve as a neurobiological marker of impaired

Abbreviations: AUC-ROC, areas under the receiver operating characteristic curves; BDNF, brain-derived neurotrophic factor; CGI-I, clinical global impression improvement; CGI-S, clinical global impression severity; DE, depressive episode; DSM-5, Diagnostic and Statistical Manual of Mental Disorders, Fifth Edition; DSST, digit symbol substitution test; IGF-1, insulinlike growth factor-1; HC, healthy controls; MADRS, Montgomery-Asberg depression rating scale; MDD, major depressive disorder; PDQ-5, perceived deficit questionnaire-5; RAVLT, Rey auditory verbal learning test; ROC, receiver-operating characteristic; SD, standard deviation; SPSS, statistical package for the social sciences; TMT-B, trail making test $\mathrm{B}$. neuroplastic processes in MDD. In addition, the increase of the neurotrophin concentration could indicate therapy effectiveness.

According to our recent systematized data, there are discrepancies in IGF-1 levels in MDD patients across the studies, although the majority demonstrate higher levels of peripheral IGF-1 compared to healthy controls (HC) (8). The elevation of peripheral total IGF-1 concentrations and its decline after antidepressants' treatment were established in several studies in MDD patients (21-23), moreover, it was reported that only patients in remission had attenuated IGF-1 concentrations following treatment $(21,22)$. The enhancement of the peripheral IGF-1 expression in MDD may be a compensatory mechanism in response to its brain synthesis decrease (24) or diminished neurotrophin bioavailability due to the hyposensitivity of IGF-1 receptors under the neuroinflammatory stress (25). In our recent work, we assumed that the activity of the cerebral-hepatic axis rise in response to inadequate cerebral IGF-1 levels (26). As a consequence, the production of IGF-1 in the liver increases. When cerebral IGF-1 production is restored, the liver IGF-1 production and its blood concentration decrease.

However, the clinical value, sensitivity, specificity, and predictive significance of a single biomarker for MDD remain doubtful. To solve this problem, the approach that uses the cumulative sensitivity and specificity of biomarkers' combination might be applicable (27). Therefore, in our study we aimed: 1) to evaluate if BDNF and IGF-1 serum concentrations separately and in combination might be biomarkers for MDD diagnosis and treatment response; 2) to evaluate the relationships between clinical MDD parameters and serum levels of the above-mentioned neurotrophins.

\section{MATERIALS AND METHODS}

\section{Study Design}

This was a case-control study, which included 73 participants aged 18 to 65 years. Outpatients $(n=41)$ with MDD diagnosis according to DSM-5 criteria (28) were included through Zaporizhzhia Regional Clinical Psychiatric Hospital, Ukraine. Eligibility criteria for the study participants were described elsewhere (29). Before entering the study, all patients had received no actual antidepressant medication. Subjects were excluded if they had any other psychiatric diagnosis, high suicidal risk, substance dependence/abuse over the past year, 
significant neurological disorders, head trauma, unstable medical conditions, history of endocrine diseases, psychotic symptoms, the risk for the hypomanic switch (29). Thirty-two healthy controls (HC) with no current psychiatric disorder were enrolled within the same period that the MDD patients were included. HC were excluded based on the use of medications and/or illicit drugs; the intake of alcohol within $48 \mathrm{~h}$ of the study visit; and the presence of an unstable medical condition, which could affect cognitive function (29).

All the participants gave written informed consent to take part in the study and attended a baseline visit to undergo the evaluations. The appraisal was reiterated in 30 patients after 8 weeks of vortioxetine treatment 10-20 mg per day.

The study was approved by the local ethics committee and performed following the ethical standards laid down in the 1964 Declaration of Helsinki and its later amendments and registered at ClinicalTrials.gov (NCT03187093).

\section{Clinical Assessments}

The severity of depression at baseline and changes after treatment were assessed with MADRS (30), Clinical Global Impression Severity (CGI-S) and Improvement (CGI-I) scales (31). Perceived Deficit Questionnaire-5 (PDQ-5), which measures perceived difficulties of cognitive functioning, was used to evaluate subjective cognitive functioning (32). Functioning was rated using the Sheehan Disability Scale (SDS) (33).

\section{Neuropsychological Assessments}

To describe neurocognitive functioning, a battery of neuropsychological tests covering the most impaired cognitive domains in MDD was administered to all participants. The tests were managed using paper and pencil. The instruments included:

1. Rey Auditory Verbal Learning Test (RAVLT), to evaluate immediate verbal memory, retroactive and proactive interference effects, delayed recall, and recognition (34);

2. Trail Making Test B (TMT-B), to assess processing speed, executive function i.e., set-shifting (34);

3. Digit Symbol Substitution Test (DSST), to assess processing speed, executive function, learning, memory, attention, and concentration (34).

The procedures of the tests were described previously (29).

\section{BDNF and IGF-1 Measurements}

In MDD patients and $\mathrm{HC}, \mathrm{BDNF}$, and IGF-1 concentrations were evaluated in the morning. Blood was taken by a venous puncture between 8:00 and 11:00 a.m. within the first 2 days after clinical assessments in 73 participants at baseline and 30 patients after 8 weeks of vortioxetine (10-20 mg per day) treatment. Blood was centrifuged at $3000 \mathrm{~g}$ for $10 \mathrm{~min}$, and the serum was stored at $-20^{\circ} \mathrm{C}$ until further processing. IGF-1 was assessed with the chemiluminescence immunoassay Immulite 2500 (Siemens AG, Germany) and Human IGF-1 Quantikine ELISA Kit (R\&D Systems Inc., Minneapolis, United States). The measurement range for IGF-1 was from 20 to $1600 \mathrm{ng} / \mathrm{ml}$. BDNF was measured using the chemiluminescence immunoassay «Sunrise» (TEKAN, Austria, GmbH) and BDNF Sandwich ELISA Kit («MilliporeChemiKineTM»). The measurement range was from 15 to 1000 $\mathrm{pg} / \mathrm{ml}$.

\section{Statistical Analysis}

Data analyses were done with the SPSS for Windows, Version 20.0 (SPSS Inc., United States). The results were presented as median (interquartile range) or means (SDs) or percentages. The statistical significance of between-group comparisons was determined using non-parametric and parametric criteria when appropriate. The relationships between demographic and clinical parameters and BDNF or IGF-1 concentrations were assessed with Spearman's or Pearson's correlation coefficient. Thereafter, the areas under the receiver operating characteristic curves (AUC-ROC) were calculated to determine if the value of serum BDNF or IGF-1 level could discriminate patients with MDD from HC. A cutoff was derived from the ROC curve with empirical optimal sensitivity and specificity. In addition, a ROC curve for the combination of two biomarkers (BDNF and IGF-1) was built to assess if this combination has a higher value to discriminate patients with MDD from HC. For this purpose, we ran a binary logistic regression to get the probability and built a ROC curve using the probability as the test variable. The statistical threshold was set at $\mathrm{p}<0.05$.

\section{RESULTS}

\section{Characteristics of Controls and MDD Patients}

Table 1 demonstrates the main demographic, psychopathological, neuropsychological, and functional characteristics of MDD and HC groups. There were no differences in age, gender, and level of education between groups. Surveyed cohorts had a higher percentage of women than men. The mean years of education in HC and MDD patients was 15 years. Although the number of people with predisposing factors (those that put a person at risk of developing MDD, which included the presence of childhood psychotrauma, persistent stress, and MDD in relatives) was higher in MDD patients, only the presence of precipitating factors (specific events or triggers to the onset of the current DE) significantly differed MDD patients from HC.

Besides the expected statistical difference in MDD patients and $\mathrm{HC}$ on MADRS and CGI-S total scores, a significant distinction in neuropsychological test performance was found between the comparison groups. MDD participants were significantly worse ( $\mathrm{p}<0.0001)$ in executive functioning (DSST, TMT-B scores), processing speed (DSST, TMT-B scores), set-shifting (TMT-B), and all parameters of verbal memory (RAVLT subtests). MDD patients also had a significantly lower level of subjective cognitive functioning as compared to HC.

After 8 weeks of vortioxetine treatment in 30 MDD patients, we revealed that the intake of the antidepressant significantly improved the clinical parameters of patients (Table 1). Thus, we 
TABLE 1 | Demographic, psychopathological, neuropsychological, functional characteristics, and serum BDNF and IGF-1 levels in healthy controls and MDD patients.

\begin{tabular}{|c|c|c|c|c|c|c|}
\hline \multirow[t]{2}{*}{ Variables } & \multirow[t]{2}{*}{$\mathrm{HC}(\mathrm{n}=32)$} & \multirow[t]{2}{*}{ MDD $(n=41)$} & \multirow[t]{2}{*}{$p$} & \multicolumn{2}{|c|}{ Treatment group $(n=30)$} & \multirow[t]{2}{*}{$p$} \\
\hline & & & & Pre-treatment & Post-treatment & \\
\hline \multicolumn{7}{|l|}{ Demographic characteristics } \\
\hline Women, n (\%) & $20(62.5)^{\mathrm{b}}$ & 27 (65.9) & 0.77 & $20(69)$ & - & \\
\hline Age, years ${ }^{\S}$ & $38.0(12.2)^{\mathrm{a}}$ & $36.4(12.8)$ & 0.60 & $35.1(12.9)$ & - & \\
\hline Education, years ${ }^{\S}$ & $15.4(1.9)^{\mathrm{a}}$ & $14.7(2.0)$ & 0.16 & $14.6(2.1)$ & - & \\
\hline Childhood psychotrauma, n (\%) & $4(12.5)^{b}$ & $13(31.7)$ & 0.05 & $10(34.5)$ & - & \\
\hline Persistent stress, n (\%) & $12(37.5)^{b}$ & $22(53.7)$ & 0.17 & $14(48.3)$ & - & \\
\hline Precipitating factors, n (\%) & $3(9.4)^{\mathrm{b}}$ & $26(63.4)^{\star \star}$ & $<0.0001$ & $20(69.0)^{\star \star}$ & & \\
\hline Number of DE & - & $1(1-2)$ & - & $1(1-2.5)$ & - & \\
\hline MDD in relatives, $\mathrm{n}(\%)$ & $4(12.5)^{b}$ & $12(29.3)$ & 0.09 & $10(34.5)$ & - & \\
\hline History of DE, n (\%) & - & $14(34.1)$ & - & $11(37.9)$ & - & \\
\hline \multicolumn{7}{|l|}{ Clinical assessments } \\
\hline \multicolumn{7}{|l|}{ Psychopathological } \\
\hline MADRS total score & $2(0-3.5)^{\mathrm{c}}$ & $28(21.5-31.5)^{\star \star}$ & $<0.0001$ & $29(24.5-33)^{d}$ & $5(2.5-10)^{\# \#}$ & $<0.0001$ \\
\hline CGI-S score & $1(1-1)^{\mathrm{C}}$ & $4(4-4.5)^{\star \star}$ & $<0.0001$ & $4(4-5)^{d}$ & $2(1-3)^{\# \#}$ & $<0.0001$ \\
\hline \multicolumn{7}{|c|}{ Patient-reported cognitive symptoms } \\
\hline PDQ-5 total score & $1(0-2)^{c}$ & $6(4-11.5)^{\star \star}$ & $<0.0001$ & $7(4-12.5)^{d}$ & $2(1-4)^{\# \#}$ & $<0.0001$ \\
\hline \multicolumn{7}{|l|}{ Neuropsychological } \\
\hline RAVLT immediate recall total & $65.5(58.25-69)^{\mathrm{c}}$ & $50.5(45-54.5)^{\star \star}$ & $<0.0001$ & $51(45-54)^{d}$ & $69(65-72.5)^{\# \#}$ & $<0.0001$ \\
\hline RAVLT proactive interference & $8(6-9)^{c}$ & $6(5-7)^{\star \star}$ & $<0.0001$ & $6(5-7)^{d}$ & $8(6-9)^{\# \#}$ & $<0.0001$ \\
\hline RAVLT retroactive interference & $15(13.25-15)^{\mathrm{C}}$ & $11(9-13)^{\star *}$ & $<0.0001$ & $11(9-13)^{d}$ & $15(14-15)^{\# \#}$ & $<0.0001$ \\
\hline RAVLT delayed recall & $15(13-15)^{c}$ & $11(9-13)^{\star *}$ & $<0.0001$ & $11(9-13)^{d}$ & $15(14-15)^{\# \#}$ & $<0.0001$ \\
\hline RAVLT delayed recognition & $15(15-15)^{\mathrm{c}}$ & $15(14-15)^{\star}$ & 0.003 & $15(13.5-15)^{d}$ & $15(15-15)^{\#}$ & 0.001 \\
\hline TMT-B $(\mathrm{s})^{\S}$ & $52.4(12.8)^{\mathrm{a}}$ & $72.3(21.2)^{\star \star}$ & $<0.0001$ & $72.4(23.0)^{\mathrm{e}}$ & $45.4(15.6)^{\# \#}$ & $<0.0001$ \\
\hline DSST & $63(55.5-68)^{c}$ & $50.5(44.25-59.75)^{\star \star}$ & $<0.0001$ & $54(44.5-60.5)^{d}$ & $62(52-73)^{\# \#}$ & $<0.0001$ \\
\hline \multicolumn{7}{|l|}{ Patient-rated functioning } \\
\hline SDS Work score & $0(0-0)^{c}$ & $6(5-8)^{\star \star}$ & $<0.0001$ & $6(5-8)^{d}$ & $1(0-3)^{\# \#}$ & $<0.0001$ \\
\hline SDS Social score & $0(0-0)^{c}$ & $7(3.75-9.25)^{\star \star}$ & $<0.0001$ & $7(4.5-10)^{d}$ & $1(0-3)^{\# \#}$ & $<0.0001$ \\
\hline SDS Family score & $0(0-0)^{c}$ & $6(4-8)^{\star *}$ & $<0.0001$ & $6(4.5-8)^{d}$ & $1(0-2.5)^{\# \#}$ & $<0.0001$ \\
\hline SDS Total score & $0(0-1.75)^{\mathrm{c}}$ & $19(12-24.5)^{\star *}$ & $<0.0001$ & $19.5(13.25-25)^{d}$ & $2.5(0-8)^{\# \#}$ & $<0.0001$ \\
\hline SDS absenteeism, days & $0(0-0)^{c}$ & $0(0-1)^{\star *}$ & $<0.0001$ & $0(0-1)^{d}$ & $0(0-0)^{\#}$ & 0.007 \\
\hline SDS presenteism, days & $0(0-0)^{c}$ & $3(2-5)^{\star \star}$ & $<0.0001$ & $4(2.25-5.75)^{d}$ & $0(0-1.5)^{\# \#}$ & $<0.0001$ \\
\hline \multicolumn{7}{|l|}{ Serum protein levels } \\
\hline BDNF $(\mathrm{pg} / \mathrm{ml})^{\S}$ & $853.0(93.9)^{a}$ & $727.6(87.9)^{\star \star}$ & $<0.0001$ & $737.3(90.4)^{\mathrm{e}}$ & $905.3(59.6)^{\# \# *}$ & $<0.0001$ \\
\hline IGF-1 serum level (ng/ml) ${ }^{\S}$ & $170.2(58.2)^{a}$ & $289.2(125.3)^{\star \star}$ & $<0.0001$ & $288.2(132.6)^{\mathrm{e}}$ & $173.4(71.2)^{\# \#}$ & $<0.0001$ \\
\hline
\end{tabular}

Data are presented as median (upper-lower quartile) unless otherwise stated; ${ }^{\S}$ data are presented as means (SD).

BDNF, brain-derived neurotrophic factor; CGI-S, clinical global impression-severity of illness; DE, depressive episode; DSST, digit symbol substitution test; HC, healthy controls; IGF-1, insulin-like growth factor; MADRS, Montgomery-Asberg depression rating scale; MDD, patients with major depressive disorder; PDQ-5, perceived deficits questionnaire-5 items; RAVLT, Rey auditory verbal learning test; SDS, Sheehan disability scale; TMT-B, trail making test part B.

${ }^{a}$ ANOVA (analysis of variance) analysis, controls vs. MDD patients.

${ }^{b}$ Chi-square test, controls vs. MDD patients.

${ }^{c}$ Mann-Whitney U-test, controls vs. MDD patients.

"Wilcoxon test (paired samples), "Pre-treatment" vs. "Post-treatment".

ePaired-samples t-test, "Pre-treatment" vs. "Post-treatment".

Compared with controls, ${ }^{*} p<0.05,{ }^{* *} p<0.01$. Compared with "Pre-treatment", ${ }^{\#} p<0.05,{ }^{\# \#} p<0.0001$.

observed a significant decrease in depression severity (MADRS, CGI-S, SGI-I scores), improvement of cognitive impairment (measured as subjectively as objectively), and functioning.

\section{Serum Concentrations of BDNF and IGF-1}

Table 1 shows serum protein levels of BDNF and IGF-1 in HC and MDD patients. It was demonstrated that serum BDNF concentrations were significantly lower in MDD persons compared to HC ( $p<0.0001)$, whereas the concentrations of IGF-1 were significantly higher in patients than HC $(\mathrm{p}<0.0001)$. Although IGF-1 concentrations were slightly higher in women than in men as in MDD [299.4 (131.5) ng/ml vs. 271.5 (116.5)] as in HC group [174.1 (53.4) vs. 164.2 (67.2)], those differences did not reach a significant level.
After 8 weeks of treatment with vortioxetine, BDNF levels were significantly higher in post-treatment than pre-treatment $(\mathrm{p}<$ $0.0001)$, moreover, they were prominently higher than in $\mathrm{HC}(\mathrm{F}=$ $9.36, \mathrm{p}=0.003)$. Whereas, IGF-1 concentrations in MDD group post-treatment were significantly lower than pretreatment $(\mathrm{p}<$ $0.0001)$ and not significantly different from $\mathrm{HC}(\mathrm{F}=1.86, \mathrm{p}=0.18)$.

\section{Correlations of Serum BDNF and IGF-1 Levels With Clinical Variables}

Next, we performed correlational analyses to determine possible associations between serum BDNF and IGF-1 concentrations and demographic and clinical parameters in the whole sample (Table 2). We established prominent inverse relationships between BDNF concentrations and MDD status $(\mathrm{r}=-0.57, \mathrm{p}<$ 
TABLE 2 | Spearman's/Pearson's correlations between demographic, psychopathological, neuropsychological characteristics, and serum BDNF and IGF-1 levels in MDD patients and healthy controls.

\begin{tabular}{|c|c|c|c|c|}
\hline Variables & $\begin{array}{l}\text { BDNF } \\
\text { (pg/ml) }\end{array}$ & $p$ & $\begin{array}{c}\text { IGF-1 } \\
\text { (ng/ml) }\end{array}$ & $p$ \\
\hline \multicolumn{5}{|l|}{ Demographic characteristics } \\
\hline Age, years & 0.02 & 0.9 & -0.17 & 0.16 \\
\hline Gender & 0.15 & 0.20 & 0.10 & 0.44 \\
\hline Depression status & $-0.57^{\star \star}$ & $<0.0001$ & $0.50^{\star *}$ & $<0.0001$ \\
\hline Persistent stress & 0.06 & 0.59 & 0.01 & 0.93 \\
\hline Precipitating factors & $-0.33^{\star \star}$ & 0.004 & 0.20 & 0.11 \\
\hline Recurrence of DE & -0.03 & 0.79 & 0.16 & 0.19 \\
\hline Number of DE & -0.04 & 0.75 & $0.43^{\star \star}$ & 0.001 \\
\hline Duration of DE, weeks & -0.02 & 0.85 & $0.37^{\star \star}$ & 0.002 \\
\hline MDD in relatives & -0.12 & 0.32 & 0.05 & 0.68 \\
\hline \multicolumn{5}{|l|}{ Clinical assessments } \\
\hline CGI-S score & $-0.44^{\star \star}$ & $<0.0001$ & $0.45^{\star \star}$ & $<0.0001$ \\
\hline PDQ-5 score & -0.19 & 0.16 & $0.43^{\star \star}$ & $<0.0001$ \\
\hline MADRS total score & $-0.43^{\star *}$ & $<0.0001$ & $0.46^{\star \star}$ & $<0.0001$ \\
\hline RAVLT immediate recall total score & $0.33^{\star \star}$ & 0.007 & $-0.42^{\star \star}$ & 0.001 \\
\hline RAVLT proactive interference score & 0.19 & 0.11 & $-0.40^{\star \star}$ & 0.001 \\
\hline RAVLT retroactive interference score & 0.17 & 0.16 & $-0.42^{\star \star}$ & 0.001 \\
\hline RAVLT delayed recall score & 0.23 & 0.06 & $-0.37^{\star \star}$ & 0.003 \\
\hline RAVLT delayed recognition score & 0.18 & 0.14 & -0.19 & 0.15 \\
\hline TMT-B (s) & $-0.27^{\star}$ & 0.03 & $0.55^{\star \star}$ & $<0.0001$ \\
\hline DSST score & $0.24^{\star}$ & 0.048 & $-0.45^{\star \star}$ & $<0.0001$ \\
\hline SDS Work score & $-0.37^{\star *}$ & 0.003 & $0.40^{\star \star}$ & 0.001 \\
\hline SDS Social score & $-0.46^{\star *}$ & $<0.0001$ & $0.39 * \star$ & 0.002 \\
\hline SDS Family score & $-0.38^{\star *}$ & 0.002 & $0.40^{* *}$ & 0.001 \\
\hline SDS Total score & $-0.43^{\star \star}$ & $<0.0001$ & $0.43^{\star \star}$ & $<0.0001$ \\
\hline SDS absenteism, days & -0.12 & 0.35 & 0.23 & 0.07 \\
\hline SDS presenteism, days & $-0.36^{\star \star}$ & 0.003 & $0.31^{*}$ & 0.01 \\
\hline \multicolumn{5}{|l|}{ Serum proteins`levels } \\
\hline Serum BDNF level & 1 & - & -0.17 & 0.18 \\
\hline Serum IGF-1 level & -0.17 & 0.18 & 1 & - \\
\hline
\end{tabular}

$B D N F$, brain-derived neurotrophic factor; CGI-S, clinical global impression-severity of illness; DE, depressive episode; DSST, digit symbol substitution test; HC, healthy controls; IGF-1, insulin-like growth factor; MADRS, Montgomery-Asberg depression rating scale; $M D D$, patients with major depressive disorder; $P D Q-5$, perceived deficits questionnaire5 items; RAVLT, Rey auditory verbal learning test; SDS, Sheehan disability scale; TMT-B, trail making test part $B$.

${ }^{*} p<0.05$ and ${ }^{* *} p<0.01$.
$0.01)$, precipitating factors $(\mathrm{r}=-0.33, \mathrm{p}<0.01)$, CGI-S $(\mathrm{r}=-0.44$, $\mathrm{p}<0.01)$, MADRS $(\mathrm{r}=-0.43, \mathrm{p}<0.01)$, TMT-B score $(\mathrm{r}=-0.27, \mathrm{p}<$ 0.05 ), all subdomains of functioning and positive correlations between serum BDNF levels and RAVLT immediate recall level $(\mathrm{r}=0.33, \mathrm{p}<0.01)$.

As for IGF-1, positive correlations were found between IGF-1 level and MDD status $(\mathrm{r}=0.50, \mathrm{p}<0.01)$, number $(\mathrm{r}=0.43, \mathrm{p}<$ $0.01)$ and duration of $\mathrm{DE}(\mathrm{r}=0.37, \mathrm{p}<0.01)$, CGI-S score $(\mathrm{r}=$ $0.45, \mathrm{p}<0.01)$, PDQ-5 score $(\mathrm{r}=0.43, \mathrm{p}<0.01)$, MADRS score $(\mathrm{r}=$ $0.46, \mathrm{p}<0.01)$, TMT-B score $(\mathrm{r}=0.55, \mathrm{p}<0.01)$ and all subdomains of functioning and negative associations between IGF-1 and the performance of RAVLT and DSST tests. Moreover, the correlations between IGF-1 concentrations and performance of cognitive tests were higher than that of BDNF.

\section{Serum BDNF and IGF-1 Levels for MDD Diagnosis}

The discriminating ability of serum proteins' level to separate MDD participants from $\mathrm{HC}$ was determined with ROC analysis. The diagnostic value of BDNF and IGF-1 is shown in Figure 1. Separately BDNF (cutoff $<763.17 \mathrm{pg} / \mathrm{ml}$, sensitivity $=81 \%$, specificity $=73 \%$ ) and IGF-1 (cutoff $>178.00 \mathrm{ng} / \mathrm{ml}$, sensitivity = $84 \%$, specificity $=64 \%)$ demonstrated good diagnostic effectiveness, with AUC of 0.840 and 0.824 , respectively. However, the combination of two neurotrophins showed excellent diagnostic value for MDD diagnosis, with an AUC of 0.916 .

\section{DISCUSSION}

This is the first study to investigate the diagnostic power of the combination of two biomarkers-BDNF and IGF-1-for MDD diagnosis. In our study, we found significantly lower BDNF and higher IGF-1 serum concentrations in patients compared with HC. Following 8 weeks of vortioxetine therapy, serum BDNF

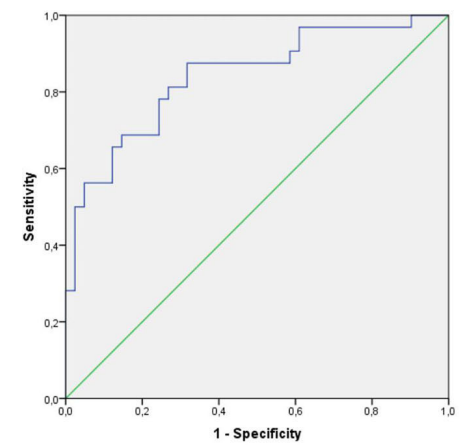

A

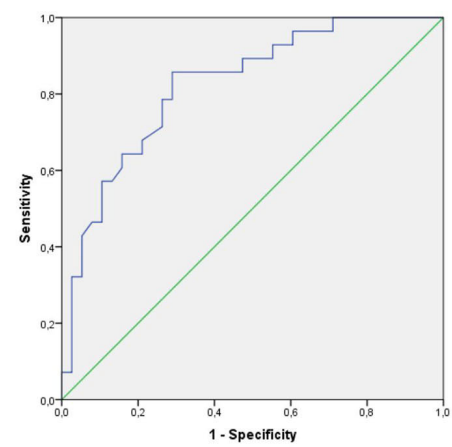

B

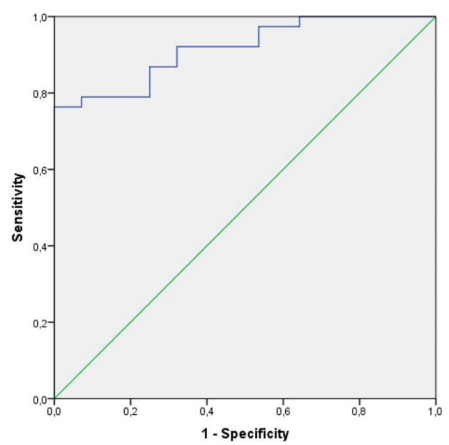

C

FIGURE 1 | Diagnostic value of serum BDNF and IGF-1 for MDD diagnosis. (A) ROC curve for BDNF to diagnose MDD ( $p<0.0001$, AUC: 0.840 ; sensitivity; $81 \%$ and specificity: $73 \%$ for a cutoff of < $763.17 \mathrm{pg} / \mathrm{ml}$ ). (B) ROC curve for IGF-1 to diagnose MDD ( $p<0.0001$, AUC: 0.824; sensitivity; $84 \%$ and specificity: 64\% for a cutoff of $>178.00 \mathrm{ng} / \mathrm{ml})$. (C) ROC curve the combination of BDNF and IGF-1 to diagnose MDD ( $p<0.0001$, AUC: 0.916). AUC, Area Under the ROC Curve; BDNF, Brain-Derived Neurotrophic Factor; IGF-1, Insulin-Like Growth Factor; MDD, major depressive disorder; ROC, receiver-operating characteristic. 
levels were significantly higher in post-treatment than pretreatment and compared to the controls. IGF-1 concentrations in subjects post-treatment were significantly lower than pretreatment and not different from the controls. Significant correlations were obtained between serum BDNF levels and MDD status, the severity of the current DE, presence of precipitating factors, executive functions' disruption (TMT-B and RAVLT immediate recall scores), and all subdomains of functioning. As for IGF-1, correlations were found between IGF1 level and MDD status, severity, number, and duration of current DE, subjective and objective cognitive functioning (PDQ-5, RAVLT subtests, TMT-B, DSST scores) and all subdomains of functioning. The associations between IGF-1 concentrations and the performance of cognitive tests were stronger than that of BDNF. Separately both BDNF and IGF-1 demonstrated good discriminating ability for MDD diagnosis; however, the combination of these two proteins had excellent diagnostic power to discriminate MDD patients from $\mathrm{HC}$, providing the AUC of 0.916 .

Firstly, we revealed that MDD patients had decreased BDNF concentrations and that these concentrations returned to normal after vortioxetine intake. These results are in line with previous works that found lower serum and plasma BDNF concentrations in depressed persons in comparison with healthy subjects $(13,14$, $17,27,35-42)$. Several antidepressants were shown to increase serum/plasma BDNF levels in MDD persons compared to pretreatment levels, including agomelatine (41), duloxetine (27), escitalopram $(27,43,44)$, fluoxetine $(41,45)$, milnacipran (36), paroxetine $(36,38,44)$, sertraline $(44)$, venlafaxine (44), and vortioxetine $(17,46)$. However, recent works have shown that not all antidepressants increase BDNF concentrations (47), and antidepressant-induced rise in BDNF concentrations are more substantial in responders compared to non-responders (48-51). Moreover, it was demonstrated that high-intensity exercises (52), electroconvulsive therapy $(15,18,19,53)$, and deep brain stimulation (54) also increase BDNF levels. In animal studies, it has been revealed that vortioxetine enhances the behaviors of depressed rodents probably by influencing the cAMP/CREB/ $\mathrm{BDNF}$ signal pathway and promoting the expression of BDNF in the dorsal and ventral hippocampus of the animals $(55,56)$. Moreover, it was shown that vortioxetine, but not fluoxetine, raised hippocampal BDNF concentrations in rats (57). Another study demonstrated that vortioxetine amplified the number of synapses and mitochondria substantially, together with increased BDNF levels, while fluoxetine showed no effect (58). It was suggested that fast changes in BDNF concentrations and synaptic/mitochondria plasticity of the hippocampus during vortioxetine treatment may be attributed to vortioxetine's modulation of 5-HT receptors (58). Longitudinal studies in humans revealed that it is more likely that BDNF is a biomarker for the state of MDD and treatment response rather than a risk factor for $\operatorname{MDD}(15,40)$.

In our study, we obtained significant associations between serum BDNF levels and MDD severity, precipitating factors, executive functions' disruption (performance of TMT-B and RAVLT immediate recall), and all subdomains of functioning. Previous studies in MDD patients have found correlations between BDNF levels and the presence of melancholic features and psychomotor retardation (59), DE severity $(36,60)$ and duration (60), and cognitive decline during performances of TMT-B (61) and verbal delayed recall (62). Nevertheless, one study evidenced no correlations between patients' plasma BDNF levels and cognitive functioning (38).

Regarding peripheral IGF-1 increase in MDD patients and its decrease after vortioxetine treatment, our results are consistent with some studies $(21,22,24,25,63-65)$. Data on a significant decrease of serum IGF-1 concentrations during antidepressant treatment are supported by previous investigations, in which amitriptyline, doxepin, fluoxetine, and paroxetine led to a substantial decline of peripheral IGF-1 levels $(21,22,66)$. Nevertheless, one study showed that free IGF-1, not total, concentrations did not change after antidepressant treatment (22). The authors suggested that discrepancies between total and biologically active free IGF-1 levels could be due to adaptation mechanisms to changes typically found in major depression (22).

Although in our study we found no significant differences in IGF-1 levels between women and men in both MDD and HC groups, several previous studies have shown variability across the genders in IGF-1 levels in MDD patients (67-69) These differences between genders may be explained by variations of sex hormone or fluctuations of growth hormone and IGF-1binding protein (67).

The reported here correlations between IGF-1 level and severity, number, and duration of DE, subjective and objective cognitive functioning (PDQ-5, RAVLT subtests, TMT-B, DSST), all subdomains of functioning are in line with our previous results (23). Nevertheless, here we pointed out that the associations for IGF-1 concentrations and the level of performance of cognitive tests were stronger than those for BDNF levels.

Lastly, the results of ROC-analysis demonstrated that BDNF and IGF-1 separately provided fairly good diagnostic power to separate MDD patients from $\mathrm{HC}$, meanwhile the combination of these neurotrophins showed excellent diagnostic value. Therefore, serum BDNF and IGF-1 levels might be a potential biomarker combination as a diagnostic test for MDD. To the best of our knowledge, we were the first to combine those two serum proteins for MDD diagnosis. Previously it was shown that the arrangement of tPA, BDNF, TrkB, proBDNF, and p75NTR might be a diagnostic biomarker panel for MDD (27) and the combination of BDNF, FGF-2, TNF- $\alpha$, and 5-HT may predict the efficacy of escitalopram therapy (70).

\section{Limitations}

Our study was limited by its small sample size, therefore, obtained data need to be replicated in larger samples to confirm our findings.

\section{CONCLUSIONS}

Patients with MDD had significantly lower BDNF and higher IGF-1 serum concentrations compared to controls. Vortioxetine 
treatment normalized those disruptions, moreover, after 8 weeks of treatment BDNF concentrations in MDD individuals were significantly higher compared to HC. In the whole sample at baseline BDNF levels correlated with the presence of precipitating factors, meanwhile, IGF-1 levels-with the number and duration of DE. Therefore, we can suggest that $\mathrm{BDNF}$ is related to acute stress. As for cognition, IGF-1 had stronger associations than BDNF with the disturbances in different cognitive domains, also, it correlated with the subjective cognitive level. Both factors were associated with functioning in different spheres of life. As BDNF as IGF-1 were significantly associated with MDD status and severity of a current episode and separately demonstrated good discriminating ability for MDD diagnosis. However, their combination had excellent diagnostic power to discriminate MDD patients from HC. Thus, the combination of IGF-1 and BDNF might be considered as a biomarker panel for MDD diagnosis.

\section{DATA AVAILABILITY STATEMENT}

The datasets generated for this study are available on request to the corresponding authors.

\section{REFERENCES}

1. Macaluso M, Preskorn SH. How biomarkers will change psychiatry: from clinical trials to practice. Part I: introduction. J Psychiatr Pract (2012) 18 (2):118-21. doi: 10.1097/01.pra.0000413277.11091.25

2. Kalia M, Costa E Silva J. Biomarkers of psychiatric diseases: current status and future prospects. Metabolism (2015) 64(3 Suppl 1):S11-5. doi: 10.1016/ j.metabol.2014.10.026

3. Venkatasubramanian G, Keshavan MS. Biomarkers in Psychiatry - A Critique. Ann Neurosci (2016) 23(1):3-5. doi: 10.1159/000443549

4. Liu B, Liu J, Wang M, Zhang Y, Li L. From Serotonin to Neuroplasticity: Evolvement of Theories for Major Depressive Disorder. Front Cell Neurosci (2017) 28(11):305. doi: 10.3389/fncel.2017.00305

5. Boku S, Nakagawa S, Toda H, Hishimoto A. Neural basis of major depressive disorder: Beyond monoamine hypothesis. Psychiatry Clin Neurosci (2018) 72 (1):3-12. doi: $10.1111 / p c n .12604$

6. Duman RS. Role of neurotrophic factors in the etiology and treatment of mood disorders. Neuromol Med (2004) 5(1):11-25. doi: 10.1385/NMM:5:1:011

7. Phillips C. Brain-Derived Neurotrophic Factor, Depression, and Physical Activity: Making the Neuroplastic Connection. Neural Plast (2017) 2017:7260130. doi: 10.1155/2017/7260130

8. Levada OA, Troyan AS. Insulin-like growth factor-1: a possible marker for emotional and cognitive disturbances, and treatment effectiveness in major depressive disorder. Ann Gen Psychiatry (2017) 26(16):38. doi: 10.1186/ s12991-017-0161-3

9. Mitre M, Mariga A, Chao MV. Neurotrophin signalling: novel insights into mechanisms and pathophysiology. Clin Sci (Lond) (2017) 131(1):13-23. doi: 10.1042/CS20160044

10. Guilloux JP, Douillard-Guilloux G, Kota R, Wang X, Gardier AM, Martinowich K, et al. Molecular evidence for BDNF- and GABA-related dysfunctions in the amygdala of female subjects with major depression. $\mathrm{Mol}$ Psychiatry (2012) 17(11):1130-42. doi: 10.1038/mp.2011.113

11. Hayley S, Du L, Litteljohn D, Palkovits M, Faludi G, Merali Z, et al. Gender and brain regions specific differences in brain derived neurotrophic factor protein levels of depressed individuals who died through suicide. Neurosci Lett (2015) 23(600):12-6. doi: 10.1016/j.neulet.2015.05.052

\section{ETHICS STATEMENT}

The studies involving human participants were reviewed and approved by the local ethics committee of State Institution Zaporizhzhia Medical Academy of Postgraduate Education Ministry of Health of Ukraine. The patients/participants provided their written informed consent to participate in this study.

\section{AUTHOR CONTRIBUTIONS}

OL supervised the study. OL and AT were involved in the concept of the study and collection of the data. Both authors equally contributed to data preparation and literature search. OL and AT analyzed the data and wrote the first draft of the manuscript. All authors contributed to the article and approved the submitted version. of the manuscript.

\section{ACKNOWLEDGMENTS}

We are grateful to Viktoria V. Levada for substantial help in data collection.

12. Dunham JS, Deakin JF, Miyajima F, Payton A, Toro CT. Expression of hippocampal brain-derived neurotrophic factor and its receptors in Stanley consortium brains. J Psychiatr Res (2009) 43(14):1175-84. doi: 10.1016/ j.jpsychires.2009.03.008

13. Molendijk ML, Spinhoven P, Polak M, Bus BA, Penninx BW, Elzinga BM. Serum BDNF concentrations as peripheral manifestations of depression: evidence from a systematic review and meta-analyses on 179 associations (N=9484). Mol Psychiatry (2014) 19(7):791-800. doi: 10.1038/mp.2013.105

14. Levada OA, Cherednichenko NV. Brain-derived neurotrophic factor (BDNF): neurobiology and marker value in neuropsychiatry. Lik Sprava (2015) 3-4:15-25.

15. Kishi T, Yoshimura R, Ikuta T, Iwata N. Brain-Derived Neurotrophic Factor and Major Depressive Disorder: Evidence from Meta-Analyses. Front Psychiatry (2018) 8:308. doi: 10.3389/fpsyt.2017.00308

16. Matrisciano F, Bonaccorso S, Ricciardi A, Scaccianoce S, Panaccione I, Wang L, et al. Changes in BDNF serum levels in patients with major depression disorder (MDD) after 6 months treatment with sertraline, escitalopram, or venlafaxine. J Psychiatr Res (2009) 43(3):247-54. doi: 10.1016/j.jpsychires.2008.03.014

17. Sagud M, Nikolac Perkovic M, Vuksan-Cusa B, Maravic A, Svob Strac D, Mihaljevic Peles A, et al. A prospective, longitudinal study of platelet serotonin and plasma brain-derived neurotrophic factor concentrations in major depression: effects of vortioxetine treatment. Psychopharmacol (Berl) (2016) 233(17):3259-67. doi: 10.1007/s00213-016-4364-0

18. Brunoni AR, Baeken C, Machado-Vieira R, Gattaz WF, Vanderhasselt MA BDNF blood levels after electroconvulsive therapy in patients with mood disorders: a systematic review and meta-analysis. World J Biol Psychiatry (2014) 15(5):411-8. doi: 10.3109/15622975.2014.892633

19. Rocha RB, Dondossola ER, Grande AJ, Colonetti T, Ceretta LB, Passos IC, et al. Increased BDNF levels after electroconvulsive therapy in patients with major depressive disorder: A meta-analysis study. J Psychiatr Res (2016) 83:47-53. doi: 10.1016/j.jpsychires.2016.08.004

20. Kallies G, Rapp MA, Fydrich T, Fehm L, Tschorn M, Terán C, et al. Serum brain-derived neurotrophic factor (BDNF) at rest and after acute aerobic exercise in major depressive disorder. Psychoneuroendocrinology (2019) 102:212-5. doi: 10.1016/j.psyneuen.2018.12.015

21. Deuschle M, Blum WF, Strasburger CJ, Schweiger U, Weber B, Körner A, et al. Insulin-like growth factor-I (IGF-I) plasma concentrations are increased in 
depressed patients. Psychoneuroendocrinology (1997) 22:493-503. doi: 10.1016/S0306-4530(97)00046-2

22. Weber-Hamann B, Blum WF, Kratzsch J, Gilles M, Heuser I, Deuschle M. Insulin-like growth factor-I (IGF-I) serum concentrations in depressed patients: relationship to saliva cortisol and changes during antidepressant treatment. Pharmacopsychiatry (2009) 42:23-8. doi: 10.1055/s-0028-1085442

23. Levada OA, Troyan AS, Pinchuk IY. Serum insulin-like growth factor-1 as a potential marker for MDD diagnosis, its clinical characteristics, and treatment efficacy validation: data from an open-label vortioxetine study. BMC Psychiatry (2020) 20:208. doi: 10.1186/s12888-020-02636-7

24. Bot M, Milaneschi Y, Penninx BW, Drent ML. Plasma insulin-like growth factor I levels are higher in depressive and anxiety disorders, but lower in antidepressant medication users. Psychoneuroendocrinology (2016) 68:14855. doi: 10.1016/j.psyneuen.2016.02.028

25. Tu KY, Wu MK, Chen YW, Lin PY, Wang HY, Wu CK, et al. Significantly Higher Peripheral Insulin-Like Growth Factor-1 Levels in Patients With Major Depressive Disorder or Bipolar Disorder Than in Healthy Controls: A Meta-Analysis and Review Under Guideline of PRISMA. Med (Baltimore) (2016) 95(4):e2411. doi: 10.1097/MD.0000000000002411

26. Levada OA, Troyan AS. Major depressive disorder and accelerated aging from a peripheral IGF-1 overexpression perspective. Med Hypotheses (2020) 138:109610. doi: 10.1016/j.mehy.2020.109610

27. Jiang H, Chen S, Li C, Lu N, Yue Y, Yin Y, et al. The serum protein levels of the tPA-BDNF pathway are implicated in depression and antidepressant treatment. Transl Psychiatry (2017) 7(4):e1079. doi: 10.1038/tp.2017.43

28. American Psychiatric Association. Diagnostic and Statistical Manual of Mental Disorders, Fifth Edition. American Psychiatric Association: Arlington, VA (2013).

29. Levada OA, Troyan AS. Cognitive-functional relationships in major depressive disorder: Crucial data from a Ukrainian open-label study of vortioxetine versus escitalopram. J Affect Disord (2019) 250:114-22. doi: 10.1016/j.jad.2019.03.040

30. Montgomery SA, Åsberg M. A new depression scale designed to be sensitive to change. Br J Psychiatry (1979) 134:382-9. doi: 10.1192/bjp.134.4.382

31. Guy W. Clinical Global Impressions. In: Guy W, editor. ECDEU Assessment Manual for Psychopharmacology Revised. Rockville: National Institute of Mental Health (1976). p. 217-22.

32. Cha D. Perceived Deficits Questionnaire - Depression, 5-item (PDQ-D-5). In: McIntyre R, editor. Cognitive impairment in major depressive disorder: Clinical relevance, biological substrates, and treatment opportunities. Cambridge: Cambridge University Press (2016). p. 242-56.

33. Sheehan DV, Harnett-Sheehan K, Raj BA. The measurement of disability. Int Clin Psychopharmacol (1996) 11(Suppl 3):89-95. doi: 10.1097/00004850199606003-00015

34. Strauss E, Sherman EMS, Spreen O. A compendium of neuropsychological tests: Administration, norms, and commentary. Oxford University Press: Oxford, England (2006)

35. Karege F, Perret G, Bondol G, Schwald M, Bertschy G, Aubry JM. Decreased serum brain-derived neurotrophic factor levels in major depressed patients. Psychiatry Res (2002) 109(2):143-8. doi: 10.1016/S0165-1781(02)00005-7

36. Yoshimura R, Mitoma M, Sugita A, Hori H, Okamoto T, Umene W, et al. Effects of paroxetine or milnacipran on serum brain-derived neurotrophic factor in depressed patients. Prog Neuropsychopharmacol Biol Psychiatry (2007) 31(5):1034-7. doi: 10.1016/j.pnpbp.2007.03.001

37. Sen S, Duman R, Sanacora G. Serum brain-derived neurotrophic factor, depression, and antidepressant medications: meta-analyses and implications. Biol Psychiatry (2008) 64(6):527-32. doi: 10.1016/j.biopsych.2008.05.005

38. Munno D, Sterpone S, Fania S, Cappellin F, Mengozzi G, Saroldi M. Plasma brain derived neurotrophic factor levels and neuropsychological aspects of depressed patients treated with paroxetine. Panminerva Med (2013) 55 (4):377-84

39. Polyakova M, Stuke K, Schuemberg K, Mueller K, Schoenknecht P, Schroeter ML. BDNF as a biomarker for successful treatment of mood disorders: a systematic \& quantitative meta-analysis. J Affect Disord (2015) 174:432-40. doi: 10.1016/j.jad.2014.11.044

40. Bus BA, Molendijk ML. The neurotrophic hypothesis of depression. Tijdschr Psychiatr (2016) 58(3):215-22.

41. Gupta K, Gupta R, Bhatia MS, Tripathi AK, Gupta LK. Effect of Agomelatine and Fluoxetine on HAM-D Score, Serum Brain-Derived Neurotrophic Factor, and Tumor Necrosis Factor- $\alpha$ Level in Patients With Major Depressive
Disorder With Severe Depression. J Clin Pharmacol (2017) 57(12):1519-26. doi: $10.1002 / j c p h .963$

42. Schröter K, Brum M, Brunkhorst-Kanaan N, Tole F, Ziegler C, Domschke K, et al. Longitudinal multi-level biomarker analysis of BDNF in major depression and bipolar disorder. Eur Arch Psychiatry Clin Neurosci (2019) 270 (2):169-81. doi: 10.1007/s00406-019-01007-y

43. Lee BH, Park YM, Um TH, Kim S. Lower serum brain-derived neurotrophic factor levels are associated with failure to achieve remission in patients with major depression after escitalopram treatment. Neuropsychiatr Dis Treat (2014) 10:1393-8. doi: 10.2147/NDT.S64913

44. Zhou C, Zhong J, Zou B, Fang L, Chen J, Deng X, et al. Meta-analyses of comparative efficacy of antidepressant medications on peripheral BDNF concentration in patients with depression. PloS One (2017) 12(2):e0172270. doi: 10.1371/journal.pone.0172270

45. Başterzi AD, Yazici K, Aslan E, Delialioğlu N, Taşdelen B, Tot Acar S, et al. Effects of fluoxetine and venlafaxine on serum brain derived neurotrophic factor levels in depressed patients. Prog Neuropsychopharmacol Biol Psychiatry (2009) 33(2):281-5. doi: 10.1016/j.pnpbp.2008.11.016

46. Yan G, Zhang M, Liu Y, Yin M. Efficacy of vortioxetine combined cognitive behaviour intervention therapy on brain-derived neurotrophic factor level on depressive patients. Psychogeriatrics (2019) 19 (5):475-81. doi: 10.1111/psyg.12426

47. Molendijk ML, Bus BA, Spinhoven P, Penninx BW, Kenis G, Prickaerts J, et al. Serum levels of brain-derived neurotrophic factor in major depressive disorder: state-trait issues, clinical features and pharmacological treatment. Mol Psychiatry (2011) 16(11):1088-95. doi: 10.1038/mp.2010.98

48. Lee HY, Kim YK. Plasma brain-derived neurotrophic factor as a peripheral marker for the action mechanism of antidepressants. Neuropsychobiology (2008) 57(4):194-9. doi: 10.1159/000149817

49. Bocchio-Chiavetto L, Bagnardi V, Zanardini R, Molteni R, Nielsen MG, Placentino A, et al. Serum and plasma BDNF levels in major depression: a replication study and meta-analyses. World J Biol Psychiatry (2010) 11 (6):763-73. doi: 10.3109/15622971003611319

50. Kurita M, Nishino S, Kato M, Numata Y, Sato T. Plasma brain-derived neurotrophic factor levels predict the clinical outcome of depression treatment in a naturalistic study. PloS One (2012) 7(6):e39212. doi: 10.1371/journal.pone.0039212

51. Furuse K, Ukai W, Hashimoto E, Hashiguchi H, Kigawa Y, Ishii T, et al. Antidepressant activities of escitalopram and blonanserin on prenatal and adolescent combined stress-induced depression model: Possible role of neurotrophic mechanism change in serum and nucleus accumbens. J Affect Disord (2019) 247:97-104. doi: 10.1016/j.jad.2019.01.007

52. Yong KJ, Chang $\mathrm{H}$. The effect of exercise intensity on brain derived neurotrophic factor and memory in adolescents. Environ Health Prev Med (2017) 22:27. doi: 10.1186/s12199-017-0643-6

53. van Zutphen EM, Rhebergen D, van Exel E, Oudega ML, Bouckaert F, Sienaert $P$, et al. Brain-derived neurotrophic factor as a possible predictor of electroconvulsive therapy outcome. Transl Psychiatry (2019) 9(1):155. doi: 10.1038/s41398-019-0491-9

54. Dandekar MP, Saxena A, Scaini G, Shin JH, Migut A, Giridharan VV, et al. Medial Forebrain Bundle Deep Brain Stimulation Reverses Anhedonic-Like Behavior in a Chronic Model of Depression: Importance of BDNF and Inflammatory Cytokines. Mol Neurobiol (2019) 56(6):4364-80. doi: 10.1007/ s12035-018-1381-5

55. Yu H, Chen JJ, Zeng BQ, Zhong QP, Xu JP, Liu YG. Role of cAMP/CREB/ BDNF signaling pathway in anti-depressive effect of vortioxetine in mice. Nan Fang Yi Ke Da Xue Xue Bao (2017) 37(1):107-12. doi: 10.3969/j.issn.16734254.2017.01.20

56. Brivio P, Corsini G, Riva MA, Calabrese F. Chronic vortioxetine treatment improves the responsiveness to an acute stress acting through the ventral hippocampus in a glucocorticoid-dependent way. Pharmacol Res (2019) 142:14-21. doi: 10.1016/j.phrs.2019.02.006

57. Lu Y, Ho CS, McIntyre RS, Wang W, Ho RC. Effects of vortioxetine and fluoxetine on the level of Brain Derived Neurotrophic Factors (BDNF) in the hippocampus of chronic unpredictable mild stress-induced depressive rats. Brain Res Bull (2018) 142:1-7. doi: 10.1016/j.brainresbull.2018.06.007

58. Chen F, Danladi J, Ardalan M, Elfving B, Müller HK, Wegener G, et al. A Critical Role of Mitochondria in BDNF-Associated Synaptic Plasticity After One-Week Vortioxetine Treatment. Int J Neuropsychopharmacol (2018) 21 (6):603-15. doi: 10.1093/ijnp/pyy022 
59. Primo de Carvalho Alves L, Sica da Rocha N. Lower levels of brain-derived neurotrophic factor are associated with melancholic psychomotor retardation among depressed inpatients. Bipolar Disord (2018) 20(8):746-52. doi: $10.1111 /$ bdi.12636

60. Kheirouri S, Noorazar SG, Alizadeh M, Dana-Alamdari L. Elevated BrainDerived Neurotrophic Factor Correlates Negatively with Severity and Duration of Major Depressive Episodes. Cognit Behav Neurol (2016) 29 (1):24-31. doi: 10.1097/WNN.0000000000000089

61. Oral E, Canpolat S, Yildirim S, Gulec M, Aliyev E, Aydin N. Cognitive functions and serum levels of brain-derived neurotrophic factor in patients with major depressive disorder. Brain Res Bull (2012) 88(5):454-9. doi: 10.1016/j.brainresbull.2012.03.005

62. Engelmann J, Wagner S, Wollschläger D, Kaaden S, Schlicht KF, Dreimüller N, et al. Higher BDNF plasma levels are associated with a normalization of memory dysfunctions during an antidepressant treatment. Eur Arch Psychiatry Clin Neurosci (2019) 270: 183-93. doi: 10.1007/s00406-019-01006-Z

63. Lesch KP, Rupprecht R, Müller U, Pfüller H, Beckmann H. Insulin-like growth factor I in depressed patients and controls. Acta Psychiatr Scand (1988) 78:684-8. doi: 10.1111/j.1600-0447.1988.tb06404.x

64. Franz B, Buysse DJ, Cherry CR, Gray NS, Grochocinski VJ, Frank E, et al. Insulin-like growth factor 1 and growth hormone binding protein in depression: a preliminary communication. J Psychiatr Res (1999) 33:121-7. doi: 10.1016/S0022-3956(98)00066-1

65. Kopczak A, Stalla GK, Uhr M, Lucae S, Hennings J, Ising M, et al. IGF-I in major depression and antidepressant treatment response. Eur Neuropsychopharmacol (2015) 25:864-72. doi: 10.1016/j.euroneuro.2014.12.013

66. Sharma AN, da Costa e Silva BF, Soares JC, Carvalho AF, Quevedo J. Role of trophic factors GDNF, IGF-1 and VEGF in major depressive disorder: A comprehensive review of human studies. J Affect Disord (2016) 197:9-20. doi: 10.1016/j.jad.2016.02.067

67. Sievers C, Auer MK, Klotsche J, Athanasoulia AP, Schneider HJ, Nauck M, et al. IGF-I levels and depressive disorders: results from the Study of Health in Pomerania (SHIP). Eur Neuropsychopharmacol (2014) 24:890-6. doi: 10.1016/ j.euroneuro.2014.01.008

68. Emeny RT, Bidlingmaier M, Lacruz ME, Linkohr B, Peters A, Reincke M, et al. Mind over hormones: sex differences in associations of wellbeing with IGF-I, IGFBP-3 and physical activity in the KORA-age study. Exp Gerontol (2014) 59:58-64. doi: 10.1016/j.exger.2014.08.001

69. Van Varsseveld NC, Van Bunderen CC, Sohl E, Comijs HC, Penninx BW, Lips $\mathrm{P}$, et al. Serum insulin-like growth factor 1 and late-life depression: a population-based study. Psychoneuroendocrinology (2015) 54:31-40. doi: 10.1016/j.psyneuen.2015.01.014

70. Xu Y, Wei H, Zhu Y, Zhu Y, Zhang N, Qin J, et al. Potential serum biomarkers for the prediction of the efficacy of escitalopram for treating depression. $J$ Affect Disord (2019) 250:307-12. doi: 10.1016/j.jad.2019.03.008

Conflict of Interest: OL is a member of advisory and/or speaker boards of the following companies: Lundbeck, Pfizer, Acino. AT gave presentations for Lundbeck and Acino.

Copyright (c) 2020 Troyan and Levada. This is an open-access article distributed under the terms of the Creative Commons Attribution License (CC BY). The use, distribution or reproduction in other forums is permitted, provided the original author(s) and the copyright owner(s) are credited and that the original publication in this journal is cited, in accordance with accepted academic practice. No use, distribution or reproduction is permitted which does not comply with these terms. 